\title{
LA CONCENTRACIÓN PLUVIOMÉTRICA DIARIA Y LAS SECUENCIAS LLUVIOSAS EN CANARIAS: DOS FACTORES DE PELIGROSIDAD
}

\author{
Pablo Máyer Suárez \\ Departamento de Geografía. Universidad de Las Palmas de Gran Canaria \\ pmayer@dgeo.ulpgc.es \\ María Victoria Marzol Jaén \\ Departamento de Geografía. Universidad de La Laguna. \\ mmarzol@ull.es
}

\section{RESUMEN}

Los valores superiores a 0,63 del índice de concentración diaria de las precipitaciones (CI) en las 29 localidades de Canarias analizadas, evidencian uno de los rasgos más característicos de la pluviosidad: su elevada concentración. Ésta es diferente según la altitud y orientación del relieve; así, el IC es superior en las vertientes Este y Sur de las islas que en las del Norte. Este comportamiento diferente de la pluviosidad también se detecta al analizar las rachas lluviosas ya que el $40 \%$ y el $70 \%$ se producen en un solo día, durante el cual se contabiliza entre el $9 \%$ y el $35 \%$ de la precipitación anual.

Palabras clave: Índice de concentración diaria de las precipitaciones, rachas lluviosas, peligrosidad, Canarias.

\section{ABSTRACT}

The values above 0.63 in the daily precipitation concentration index (CI), in the 29 areas of the Canary Islands analyzed in this study, are evidence of one of the most characteristic features of the rainfall in the islands. However, one can see that the behaviour differs according to the altitude and orientation of the mountain slopes, in such a way that the

Fecha de recepción: julio 2012.

Fecha de aceptación: julio 2013. 
rainfall concentration is greater in the eastern and southern parts of the islands whereas the lowest indices are recorded in the north. This difference in the behaviour of the rainfall is also detected in the analysis of rainy periods.

Key words: Daily precipitation concentration index, rainy spells, risk, Canary Islands.

\section{INTRODUCCIÓN}

El análisis de la precipitación, con el objeto de caracterizar su peligrosidad a diferentes escalas espaciales y temporales, es un tema ampliamente tratado, básicamente por su implicación en los procesos geo-hidrológicos y en los análisis de erosión y pérdidas de suelo. Son varios los autores que alertan, en el contexto actual de calentamiento global, de un cambio en los patrones de la precipitación de manera que los fenómenos meteorológicos extremos ocurren cada vez con mayor frecuencia en diversos lugares del planeta como China (Zhai et al., 2005; Zhang et al., 2009; Li et al., 2011), América (Kart et al., 1998) o en diversas áreas del Mediterráneo (Coscarelli y Caloiero, 2012). En este sentido, la disminución de los días de lluvia a favor de episodios de alta intensidad que aportan importantes cantidades al total anual supone un incremento del riesgo para la población y sus actividades por una mayor frecuencia de sequías, inundaciones, deslizamientos y, en general, un mayor impacto sobre los recursos hídricos. De la misma manera, los sucesivos Paneles Intergubernamentales del Cambio Climático (IPCC's) alertan, desde hace años, de escenarios futuros en los que los totales pluviométricos descenderán, mientras que los eventos lluviosos extremos se incrementarán de manera significativa (Houghton et al., 2001; IPCC, 2012). Ello implicaría, por un lado, el aumento de la irregularidad pluviométrica con la consecuente escasez hídrica y, por otro, una mayor peligrosidad de las lluvias especialmente en regiones que, por su situación geográfica, se ven afectadas por masas de aire contrastadas -como es el caso de la península Ibérica-, o en territorios con fuertes desniveles y escasa cubierta vegetal, como es el caso de los archipiélagos atlánticos (Marzol et al., 2006; Dorta, 2007).

Es preciso, por tanto, conocer en estos ámbitos la estructura de la precipitación a escala diaria y para ello, Martín Vide (2004) propuso un Índice de Concentración de la Precipitación Diaria (CI) que ha sido aplicado en distintas regiones con resultados muy diferentes. Así, en el caso de la península Ibérica los patrones espaciales del CI muestran claramente dos ámbitos diferenciados: las regiones más próximas al Mediterráneo con índices de concentración más altos, próximos al 0,70, frente a regiones del interior peninsular o más cercanos a la vertiente atlántica donde la regularidad de la lluvia es mayor y los valores se reducen a mínimos de 0,55. Mientras que en el primer caso el 25\% de los días más lluviosos aportan casi el 80\% del total anual, en el segundo caso ese porcentaje se reduce al 65\% (Martín-Vide, 2004). En otras regiones del Mediterráneo se observan valores igualmente contrastados del IC. Coscarelli y Caloiero (2012) aplican este índice a la región de Calabria, en el sur de Italia, evidenciando los mayores valores al Este de la región, 0,62, frente a los 0,45 de la vertiente Este. Factores orográficos y otros relacionados con la dinámica atmosférica explican esas diferencias. En otras áreas, caracterizadas por una alta concentración de la lluvia como es el Sur de China, se observan valores considerablemente altos del CI. Tal es el caso de la cuenca 
de Pearl River, donde Zhang et al. (2009) muestran la distribución espacial de este índice y evidencian localidades donde el $80 \%$ de toda la lluvia se produce sólo en el $25 \%$ de los días más lluviosos.

En el caso de Canarias, donde la irregularidad y torrencialidad de las lluvias son dos de sus principales características (Marzol, 1988), este tipo de análisis es fundamental habida cuenta del incremento de los daños asociados a las precipitaciones, especialmente en las últimas décadas, y a los procesos de construcción acelerada en zonas vulnerables próximas o en los mismos cauces de los barrancos (Horcajada et al., 2000; Romero et al., 2004; Marzol et al., 2006; Dorta, 2007; Máyer y Pérez-Chacón, 2006; Máyer, 2011;).

\section{LOS DATOS UTILIZADOS}

Para el presente trabajo se escogen 91 series de precipitación diaria del período comprendido entre 1970 y 2003. Estas series han sido facilitadas por la Agencia Estatal de Meteorología y por el Servicio Hidráulico de Las Palmas. Cada serie tiene un mínimo de 30 años, no obstante, de las 91 series se eliminan 62 porque no registran correctamente la precipitación diaria, especialmente las cantidades en el rango más pequeño, de 0,1 a $0,9 \mathrm{~mm}$, bien porque no las contabilizan o bien porque esas intensidades se agrupan en un solo día. Como se observa en la figura 1, la isla que posee el mayor número de estaciones es la de Gran Canaria como consecuencia de la buena labor de mantenimiento y control efectuada, durante décadas, por el Servicio Hidráulico de Las Palmas (Ministerio de Obras Públicas). Esta gestión fue transferida, a principios de la década de los años 2000 al Consejo Insular de Aguas del Cabildo Insular de Gran Canaria.

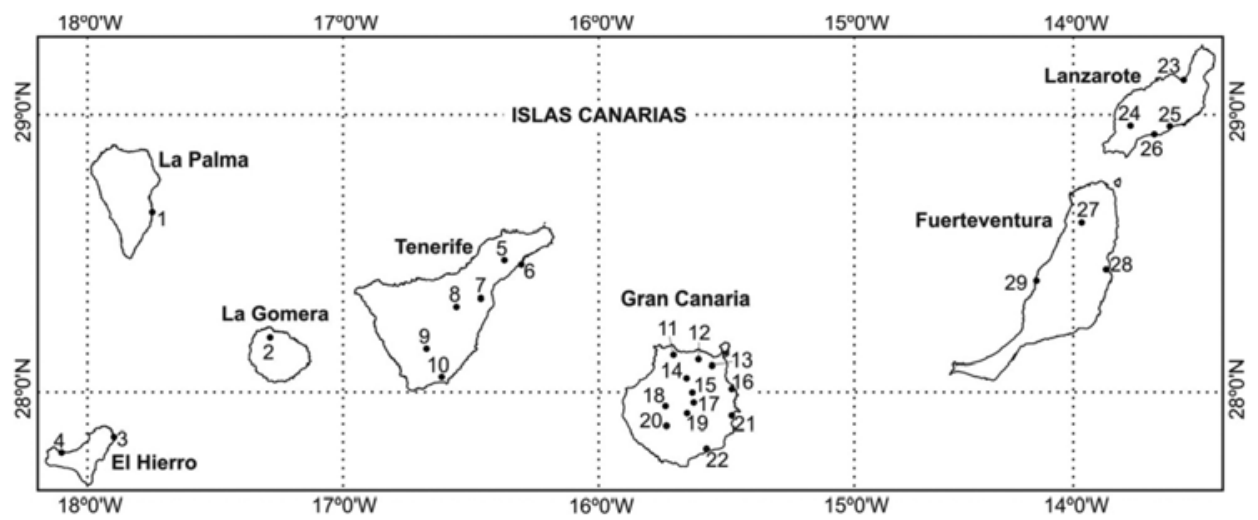

En la elección de las series es necesario señalar la importancia que tuvo su localización en el territorio, especialmente en lo que se refiere a su altitud y su orientación, factores que inducen a comportamientos contrastados de las variables climáticas en cada una de las islas. 
En las de mayor altitud, todas excepto Lanzarote y Fuerteventura, se han diferenciado tradicionalmente tres ambientes geoecológicos en función de la altitud: costa, medianía y cumbre. En general, no existe un umbral altitudinal específico para cada uno de ellos, aunque sí unos rasgos ambientales comunes que permiten adscribir cada área insular a uno de ellos. Quizás los factores más importantes a la hora de su diferenciación sean los topográficos, climáticos y edáficos que, conjuntamente, condicionan la existencia de una determinada flora e, incluso, una preferencia histórica a la hora de localizar los asentamientos de la población. Asimismo, y entre las variables topográficas, la exposición se convierte en un factor diferenciador en cada uno de los tres ambientes altitudinales, pues induce comportamientos contrastados en las variables climáticas y en el escalonamiento del paisaje vegetal (Sánchez et al., 1985). El efecto más llamativo de la distinta orientación de las vertientes es en el paisaje vegetal, pues, si bien en todas las islas se observa un escalonamiento de los pisos de vegetación, éstos varían según nos ubiquemos en las vertientes a barlovento o sotavento (Arozena, 1993). Las complicadas interacciones entre la topografía y las condiciones climáticas, así como por el paisaje vegetal resultante de ellas, hacen muy compleja una única delimitación altitudinal de esos tres ambientes generalizable a todas las islas e incluso a las vertientes opuestas de cada una de ellas. Prueba de ello, son la variedad de umbrales altitudinales utilizados por diferentes autores (Ceballos y Ortuño, 1976; Sánchez et al., 1985; Marzol, 1988; Máyer, 2011; Martín et al., 2012).

\section{OBJETIVOS Y METODOLOGÍA}

El objetivo principal de este trabajo es determinar la concentración de la precipitación diaria mediante el empleo del índice propuesto para tal fin por Martín Vide (2004). De este índice, aplicado al conjunto de la península Ibérica por Sánchez-Lorenzo y Martín Vide (2006) y a algunas regiones de la misma por De Luis et al., (1997 y 2000), se desconocen

Figura 2

CURVA DE CONCENTRACIÓN DE LA PRECIPITACIÓN DIARIA DE SANTA CRUZ DE TENERIFE Y FAMARA EN LA ISLA DE LANZAROTE (1970-2003)

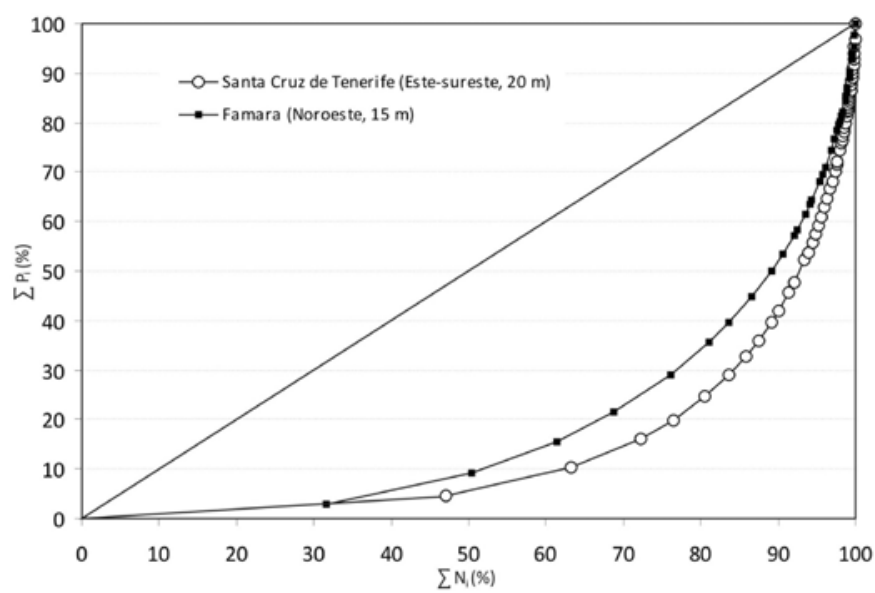


los valores que pueden alcanzar en las islas Canarias. Tal y como expone Martín Vide (2004) este índice se basa en los porcentajes del número acumulado de días de precipitación respecto al total de los días de precipitación (X), así como los porcentajes de las cantidades acumuladas de los días de precipitación con respecto a la cantidad total (Y) de las diferentes series utilizadas. Ello permite generar curvas poligonales de la concentración de la precipitación, como las representadas en la figura 2, con los datos de los valores extremos observados en dos localidades de Canarias: Santa Cruz de Tenerife y Famara en la isla de Lanzarote.

Tal y como muestra la figura 2, sobre una línea de distribución de la precipitación diaria con una regularidad perfecta, que implicaría que las cantidades de lluvia fuesen semejantes, las dos series poseen diferente separación de la curva de concentración. La mayor distancia a la línea de distribución en el caso de Santa Cruz de Tenerife supone que hay una mayor concentración de la precipitación diaria según la ecuación (1)

$$
\mathrm{Y}=\mathrm{aXebX}
$$

Las constantes a y b de esta ecuación se obtienen mediante el procedimiento de los mínimos cuadrados, de la siguiente forma (2) y (3):

$$
\begin{gathered}
\ln a=\frac{\sum \mathrm{x}_{\mathrm{i}}^{2} \sum \ln \mathrm{Y}_{\mathrm{i}}+\sum \mathrm{X}_{\mathrm{i}} \sum \mathrm{X}_{\mathrm{i}} \ln \mathrm{X}_{\mathrm{i}}-\sum \mathrm{X}_{\mathrm{i}}^{2} \sum \ln \mathrm{X}_{\mathrm{i}}-\sum \mathrm{X}_{\mathrm{i}} \sum \mathrm{X}_{\mathrm{i}} \ln \mathrm{Y}_{\mathrm{i}}}{\mathrm{N} \sum \mathrm{X}_{\mathrm{i}}^{2}-\mathrm{N}\left(\sum \mathrm{X}_{\mathrm{i}}\right)^{2}} \\
b=\frac{\mathrm{N} \sum \mathrm{X}_{\mathrm{i}} \ln \mathrm{Y}_{\mathrm{i}}+\sum \mathrm{X}_{\mathrm{i}} \sum \ln \mathrm{X}_{\mathrm{i}}-\mathrm{N} \sum \mathrm{X}_{\mathrm{i}} \ln \mathrm{X}_{\mathrm{i}}-\sum \mathrm{X}_{\mathrm{i}} \sum \ln \mathrm{Y}_{\mathrm{i}}}{\mathrm{N} \sum \mathrm{X}_{\mathrm{i}}^{2}-\left(\sum \mathrm{X}_{\mathrm{i}}\right)^{2}}
\end{gathered}
$$

siendo $\mathrm{N}$ el número de pares de valores.

Una vez determinadas las dos constantes, el cálculo de la superficie comprendida entre la recta de equidistribución y los valores observados en ambos casos nos muestra cuál de las dos series presenta una mayor concentración diaria de la precipitación. Para ello se procede al cálculo de la integral definida por la curva exponencial entre 0 y 100 de la superficie comprendida entre cada curva, el eje de abscisas y la ordenada 100 mediante la siguiente ecuación (4):

$$
A^{\prime}=\left[\frac{a}{b} e^{b x}\left(x-\frac{1}{b}\right)\right]_{0}^{100}
$$

$\mathrm{Al}$ valor obtenido mediante esta ecuación se le resta 5000 para determinar el área comprendida entre cada curva, la recta de equidistribución y la ordenada 100 (S'). De esa manera, el índice de concentración de la precipitación (CI) se obtiene de la siguiente formulación (5):

$$
C I=\frac{S^{\prime}}{5.000}
$$


El valor más elevado del índice en Santa Cruz de Tenerife $(0,71)$ implica que hay una mayor irregularidad de la precipitación diaria que en Famara $(0,63)$.

Además de obtener los valores del CI, se analizan la distribución y frecuencia de las rachas lluviosas, cuestión que permite matizar el diferente comportamiento de la pluviosidad en las distintas islas y vertientes, especialmente en unas islas donde las cuencas tienen una superficie inferior a $71 \mathrm{~km}^{2}$ y la conversión de lluvia en escorrentía se produce, en líneas generales, en muy poco tiempo (Marzol et al., 2006). Si a ello unimos una secular ocupación del territorio caracterizada por la ocupación de cauces, zonas inundables y áreas con evidencias de deslizamientos en el pasado se entienden los graves problemas que, durante los episodios de lluvia intensa, se producen en múltiples áreas urbanas de estas islas (Máyer y Pérez-Chacón, 2006).

\section{CONCENTRACIÓN PLUVIOMÉTRICA DIARIA EN CANARIAS}

La aplicación del CI exige contar con datos fiables, especialmente en la anotación, por parte de los observadores, de las cantidades de lluvia más pequeñas, es decir, aquellas de intensidad muy débil porque se recoge menos de $0,9 \mathrm{~mm}$. De otra manera se desvirtúa considerablemente el valor del IC, el cual se basa, precisamente, en la distribución de frecuencias absolutas en clases de un milímetro. Así pues, es preciso hacer una labor de depuración de los datos, comparando las estaciones más próximas para evitar utilizar aquellas que no contemplan esas intensidades y, por tanto, no se ajustan a una distribución exponencial negativa.

Como ejemplo de ese problema, se muestra, en la figura 3, la distribución de las frecuencias absolutas, en clases de un milímetro, en los primeros 30 intervalos de clases, en dos localidades situadas en la vertiente Norte de El Hierro.

Figura 3

DISTRIBUCIÓN DE FRECUENCIAS ABSOLUTAS (EN CLASES DE $1 \mathrm{~mm}$ ) DE SABINOSA Y GOLFO MERESE (EL HIERRO) EN LOS PRIMEROS 30 INTERVALOS DE CLASES

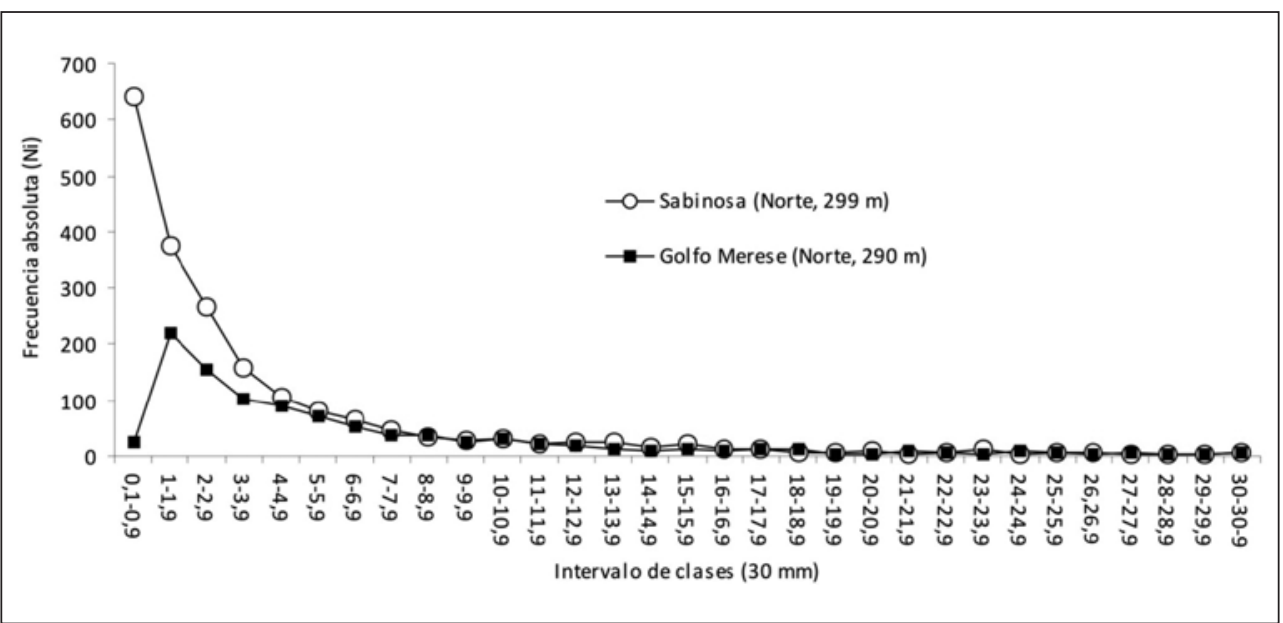


Las diferencias entre Sabinosa y Golfo Merese, en el primer intervalo son muy significativas. Mientras que en la primera localidad el número de días con precipitaciones entre 0,1 y $0,9 \mathrm{~mm}$ es de 642, en Golfo Merese apenas llega a 26 en el mismo período de 30 años.

\subsection{Relación entre $N_{i}(\%)$ y $P_{i}(\%)$}

Del análisis de los intervalos de lluvia en clases de 10 mm y su relación con los volúmenes de agua registrados en cada uno de ellos se desprenden los siguientes rasgos. En líneas generales, en Canarias las lluvias tienen un carácter débil pues el intervalo comprendido entre 1,0 y 9,9 mm es el más frecuente y agrupa, en promedio, al 55\% de los días con lluvia. Sin embargo, los volúmenes de agua recogidos con esas intensidades tienen unas variaciones muy significativas según las vertientes en las que se encuentra cada localidad. Así, en las medianías orientadas al Norte esos días con lluvias débiles contabilizan hasta el $50 \%$ de la precipitación anual, mientras que en las localidades situadas en las vertientes Sur y Oeste sólo suponen entre el $12 \%$ y $20 \%$ de la lluvia anual.

Figura 4

RELACIÓN DEL N ${ }^{\circ}$ DE DÍAS DE LLUVIA (Ni) Y LA CANTIDAD DE LLUVIA (Pi) SEGÚN SU INTENSIDAD EN DOS LOCALIDADES DE GRAN CANARIA SEGÚN LA ORIENTACIÓN
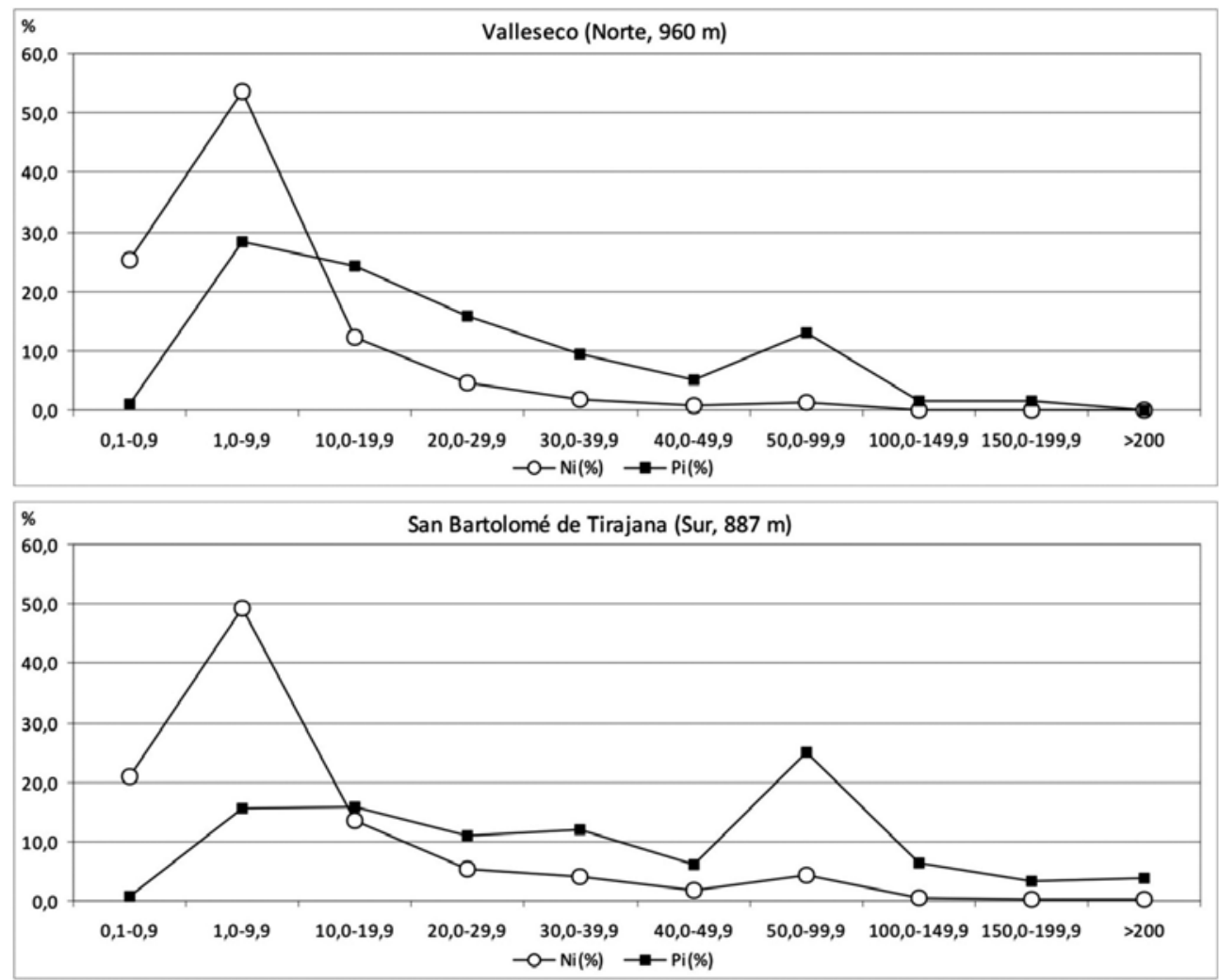
La figura 4 muestra la distribución de la precipitación en intervalos de $10 \mathrm{~mm}$ en dos localidades de la isla de Gran Canaria, San Bartolomé de Tirajana en las medianías del Sur y a 887 m.s.n.m. y Valleseco, en las del Norte a 960 m.s.n.m. Se observa que en San Bartolomé de Tirajana los mayores volúmenes de agua anual los aportan las precipitaciones considerablemente intensas, de más de $50 \mathrm{~mm}$. Este tipo de lluvias se asocian al paso de los frentes fríos de potentes perturbaciones oceánicas que, principalmente durante el invierno, afectan a esta región atlántica con vientos del tercer cuadrante -por la localización latitudinal del núcleo de la borrasca-, y que coinciden con vaguadas y depresiones aisladas en los niveles altos (D.A.N.A.S.) (Marzol et al., 2006; Dorta, 2007; Máyer, 2011). Por el contrario, en Valleseco el elevado número de días con precipitaciones débiles o muy débiles, aportan volúmenes considerables al cómputo anual de agua, y se asocian tanto a la nubosidad estratiforme propia de los alisios como al paso de los frentes con una marcada disposición Nordeste-Suroeste, que no afectan a la vertiente meridional (Máyer 2011). La diferente concentración de la precipitación también se

Figura 5

RELACIÓN DEL Nº DE DÍAS DE LLUVIA (Ni) Y LA CANTIDAD DE LLUVIA (Pi) SEGÚN SU INTENSIDAD EN DOS LOCALIDADES DE TENERIFE EN FUNCIÓN DE SU ALTITUD
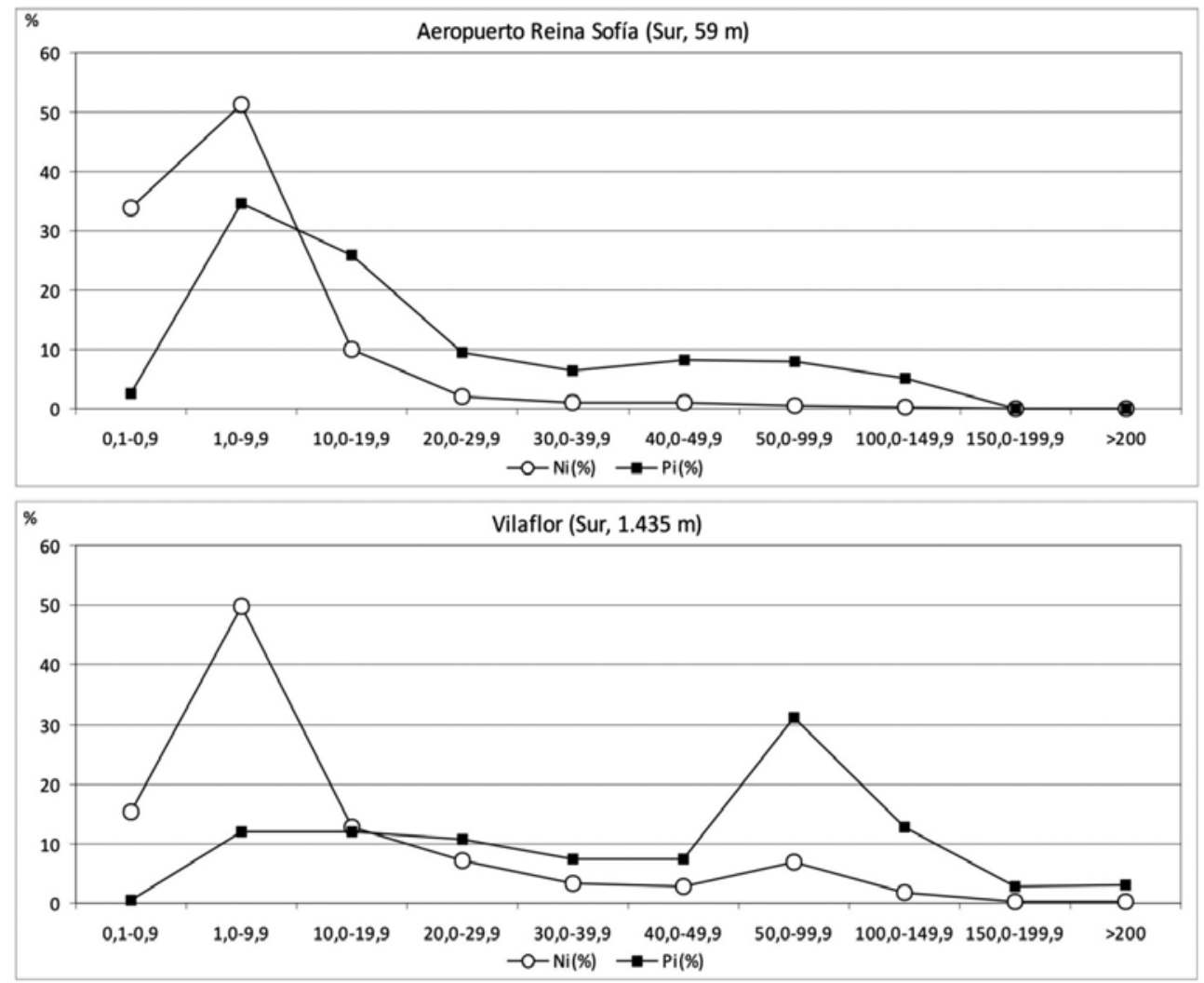
puede deducir del porcentaje que supone el $25 \%$ de los más lluviosos en el total de los días de lluvia. En San Bartolomé de Tirajana esos episodios lluviosos aportan un 80\%, mientras que en Valleseco el $77 \%$.

Por otro lado, la altitud también introduce cambios significativos en la concentración de la precipitación. Como ejemplo de ello se muestran los datos contrastados de dos localidades de la isla de Tenerife situadas en la misma vertiente pero a diferentes altitudes, el aeropuerto Reina Sofía y Vilaflor, a 59 y 1.435 m.s.n.m. respectivamente. En la figura 5 se observa cómo las lluvias con intensidades inferiores a 10,0 $\mathrm{mm}$ son especialmente frecuentes en el aeropuerto Reina Sofía, el 85\%, y aportan algo más de un tercio del volumen de agua anual; mientras que en Vilaflor esas lluvias débiles suponen el $65 \%$ de los días de lluvia y tan sólo aportan el 13\%. Por el contrario, los aguaceros más intensos -entre 50 y 150 mm/24 h- son mucho más importantes en Vilaflor que en la costa, el $9 \%$ de los días frente a $1 \%$ respectivamente. La cantidad de agua recogida en esos días tormentosos es la mitad de la lluvia anual en Vilaflor frente a sólo el 13\% en el aeropuerto. El ejemplo más destacado de ese rasgo se encuentra en las islas de Lanzarote y Fuerteventura que, por su escasa altitud, las intensidades de lluvia inferiores a 10,0 mm suponen hasta el 90\% de los días de lluvia y aportan alrededor de la mitad del volumen total de agua anual. En estas últimas islas la concentración de la precipitación es menor, y la cantidad de agua que aporta el 25\% de los días más lluviosos al total de los días es del orden del $75 \%$.

\subsection{Los valores del $\mathrm{Cl}$}

Los cambios en la concentración de la precipitación se observan de manera más evidente al obtener los valores del CI, los cuales se muestran en la tabla 1. En todas las estaciones analizadas se supera el valor de 0,63 , lo que indica una alta concentración de la precipitación. Ello supone, en líneas generales, que en Canarias el 25\% de los días más lluviosos aporta el $77 \%$ de la lluvia anual. No obstante, es necesario señalar algunas variaciones espaciales en el CI que están relacionadas con el papel del condicionante geográfico de la orientación del relieve.

Los valores más elevados del CI corresponden a las vertientes orientales y meridionales de las islas más montañosas, independientemente de la altitud a la que se encuentren. Es el caso de los aeropuertos de Mazo y Los Cangrejos, de la ciudad de Santa Cruz de Tenerife o de San Bartolomé de Tirajana y Vilaflor, todos ellos con un valor de IC muy alto -superior a 0,70-, similar al obtenido por diferentes autores en el litoral de la Comunidad valenciana (De Luis et al., 2011; Sánchez-Lorenzo y Martín Vide, 2006). Ello supone que el $80 \%$ de la lluvia proviene del $25 \%$ de los días más lluviosos. Esa elevada concentración de la lluvia tiene implicaciones directas tanto en la generación de procesos erosivos como en el origen de avenidas en los cauces de los barrancos con las consecuentes inundaciones.

En las cumbres, los valores del CI son ligeramente más bajos, de 0,66 a 0,67, como sucede en Izaña y Cuevas Blancas. Hay que señalar que en las localidades situadas por encima de 1.000 metros de altitud es frecuente que las precipitaciones tengan un carácter torrencial, de tal manera que las superiores a $50 \mathrm{~mm}$ en 24 horas representan sólo el $9 \%$ de los días con lluvia pero aportan la mitad del total de lluvia anual. 
Tabla 1

CONSTANTES a Y b DE LAS CURVAS EXPONENCIALES, COEFICIENTE DE DETERMINACIÓN $\left(R^{2}\right)$, CI Y PORCENTAJE DE LA PRECIPITACIÓN TOTAL A LA QUE CONTRIBUYE EL 25\% DE LOS DÍAS MÁS LLUVIOSOS EN LAS 29 LOCALIDADES ANALIZADAS (1970-2003)

\begin{tabular}{|c|c|c|c|c|c|c|c|c|c|}
\hline Cod & Nombre de la estación & Isla & Altitud & Orientación & a & b & $\mathbf{R}^{2}$ & CI & $\begin{array}{c}\text { Lluvia } \\
(\%)\end{array}$ \\
\hline 1 & Aeropuerto-Mazo & LP & 40 & E & 0,01082 & 0,04320 & 0,976 & 0,71 & 80,9 \\
\hline 2 & Vellehermoso & LG & 220 & $\mathrm{~N}$ & 0,02646 & 0,03493 & 0,992 & 0,64 & 74,9 \\
\hline 3 & Aeropuerto-Cangrejos & $\mathrm{EH}$ & 30 & $\mathrm{E}$ & 0,01364 & 0,04034 & 0,984 & 0,71 & 80,0 \\
\hline 4 & Sabinosa & $\mathrm{EH}$ & 270 & $\mathrm{~N}$ & 0,02041 & 0,03734 & 0,985 & 0,66 & 76,3 \\
\hline 5 & Aeropuerto-Rodeos & $\mathrm{TF}$ & 617 & $\mathrm{~N}$ & 0,02282 & 0,03674 & 0,995 & 0,64 & 74,7 \\
\hline 6 & Santa Cruz de Tenerife & $\mathrm{TF}$ & 36 & ESE & 0,00824 & 0,04627 & 0,981 & 0,71 & 80,6 \\
\hline 7 & Arafo & $\mathrm{TF}$ & 485 & $\mathrm{E}$ & 0,01631 & 0,03911 & 0,990 & 0,69 & 78,5 \\
\hline 8 & Izaña & $\mathrm{TF}$ & 2371 & CUMBRE & 0,02470 & 0,03514 & 0,989 & 0,66 & 76,3 \\
\hline 9 & Vilaflor & $\mathrm{TF}$ & 1435 & $S$ & 0,01462 & 0,04048 & 0,995 & 0,70 & 80,4 \\
\hline 10 & Aeropuerto Reina Sofía & $\mathrm{TF}$ & 59 & $S$ & 0,01892 & 0,03780 & 0,994 & 0,67 & 77,0 \\
\hline 11 & Presa de Jimenez & GC & 245 & $\mathrm{~N}$ & 0,02679 & 0,03502 & 0,991 & 0,63 & 73,5 \\
\hline 12 & Arucas & $\mathrm{GC}$ & 252 & $\mathrm{~N}$ & 0,03073 & 0,03342 & 0,993 & 0,63 & 73,6 \\
\hline 13 & Tamaraceite & $\mathrm{GC}$ & 210 & $\mathrm{~N}$ & 0,02167 & 0,03701 & 0,990 & 0,65 & 75,1 \\
\hline 14 & Valleseco & $\mathrm{GC}$ & 960 & $\mathrm{~N}$ & 0,01629 & 0,04020 & 0,993 & 0,66 & 76,7 \\
\hline 15 & Lomo Aljorradero & $\mathrm{GC}$ & 1075 & $\mathrm{NE}$ & 0,02514 & 0,03535 & 0,990 & 0,65 & 75,4 \\
\hline 16 & La Pardilla & $\mathrm{GC}$ & 45 & $\mathrm{E}$ & 0,01338 & 0,04149 & 0,979 & 0,69 & 78,2 \\
\hline 17 & Cuevas Blancas & $\mathrm{GC}$ & 1690 & CUMBRE & 0,02027 & 0,03725 & 0,991 & 0,67 & 77,0 \\
\hline 18 & Pinar de Pajonales & $\mathrm{GC}$ & 1195 & SW & 0,01529 & 0,04010 & 0,993 & 0,68 & 78,3 \\
\hline 19 & San Bartolomé de Tirajana & GC & 887 & $S$ & 0,01017 & 0,04408 & 0,995 & 0,71 & 80,4 \\
\hline 20 & Aeropuerto-Gando & $\mathrm{GC}$ & 20 & $\mathrm{E}$ & 0,01697 & 0,03899 & 0,985 & 0,68 & 77,5 \\
\hline 21 & Barranquillo Andrés & $\mathrm{GC}$ & 650 & SW & 0,01179 & 0,04306 & 0,996 & 0,69 & 78,8 \\
\hline 22 & Berriel & $\mathrm{GC}$ & 30 & SSE & 0,02088 & 0,03711 & 0,992 & 0,66 & 76,3 \\
\hline 23 & Famara & $\mathrm{LZ}$ & 15 & NW & 0,03101 & 0,03341 & 0,993 & 0,63 & 73,3 \\
\hline 24 & Yaiza & $\mathrm{LZ}$ & 155 & $\mathrm{~N}$ & 0,02881 & 0,03438 & 0,996 & 0,63 & 72,9 \\
\hline 25 & Puerto del Carmen & $\mathrm{LZ}$ & 20 & $S$ & 0,02003 & 0,03757 & 0,993 & 0,66 & 76,1 \\
\hline 26 & Guacimeta & $\mathrm{LZ}$ & 23 & $\mathrm{E}$ & 0,01988 & 0,03782 & 0,989 & 0,66 & 75,4 \\
\hline 27 & Tindaya & $\mathrm{FV}$ & 144 & $\mathrm{~N}$ & 0,02863 & 0,03429 & 0,994 & 0,63 & 73,5 \\
\hline 28 & Aeropuerto-Matorral & FV & 23 & $\mathrm{E}$ & 0,02257 & 0,03601 & 0,990 & 0,66 & 76,3 \\
\hline 29 & Puerto de la Peña & FV & 19 & W & 0,02535 & 0,03521 & 0,986 & 0,65 & 75,6 \\
\hline
\end{tabular}

La Palma (LP), La Gomera (LG), El Hierro (EH), Tenerife (TF), Gran Canaria (GC), Fuerteventura (FV) y Lanzarote (LZ).

A la vista de los resultados y de la baja correlación entre el índice CI y la altitud, se puede afirmar que esta última no es un factor de diferenciación espacial, pues tanto las zonas de costa como las medianías orientales y meridionales poseen valores igualmente altos, cuestión ésta que ya ha sido evidenciada por otros autores (De Luis et al., 1997). 
Los valores más moderados del CI en Canarias se obtienen en las vertientes N, NE y NW de las islas montañosas y en el conjunto de las de Lanzarote y Fuerteventura, donde no se supera el valor de 0,66. En el primer caso, es consecuencia del tipo de nubosidad asociada al alisio que se estanca en las vertientes septentrionales, mientras que en el segundo se explica por la menor intensidad que tienen los frentes cuando barren a las islas más orientales del archipiélago, que, además, son las de menor altitud.

\section{LA DURACIÓN Y FRECUENCIA DE LAS RACHAS LLUVIOSAS}

Un aspecto que permite matizar la concentración de la precipitación es el análisis de la duración de las rachas lluviosas y la cantidad de agua acumulada en cada una de ellas. En ese sentido, existen diferencias significativas entre las distintas islas e, incluso, entre las localidades de una misma isla en función de la longitud, la altitud y la orientación (Dávila y Romero, 1993; Marzol et al., 2006). En líneas generales, entre el 40\% y 70\% de las secuencias lluviosas se producen en un solo día, durante el cual se contabiliza entre el $9 \%$ y el 35\% de la precipitación anual.

Las localidades donde las secuencias de un solo día adquieren mayor importancia, tanto por su elevada frecuencia como por la cantidad de agua que acumulan con respecto al total anual, se encuentran en las islas más orientales, Lanzarote y Fuerteventura, y en las zonas de costa y medianías bajas de las demás islas. Así, por ejemplo, en el Puerto de la Peña, en Fuerteventura, el 70\% de las rachas lluviosas son de un solo día y en ellas se acumula el 34\% de la precipitación anual. De la misma manera, en Yaiza, Lanzarote, el 60\% de las rachas lluviosas de ese rango acumulan el $24 \%$ de la lluvia anual (fig. 6).

Figura 6

RELACIÓN ENTRE LA DURACIÓN DE LOS EPISODIOS LLUVIOSOS Y LA CANTIDAD DE LLUVIA RECOGIDA EN YAIZA (LANZAROTE)

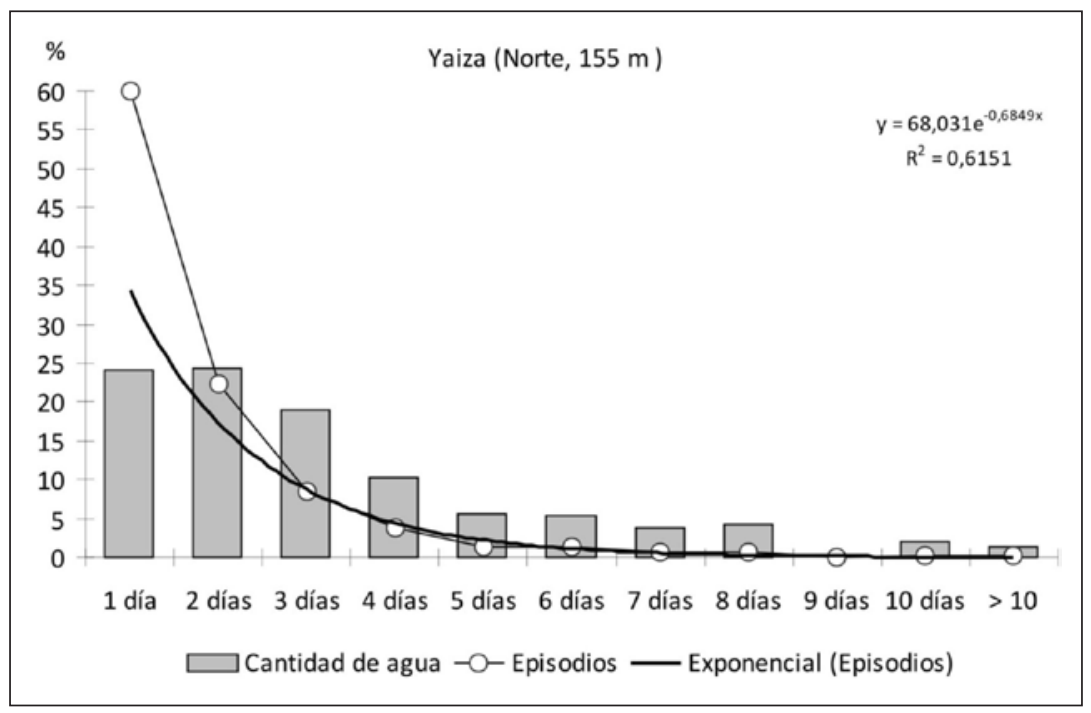


Por el contrario, las secuencias lluviosas de un solo día son poco frecuentes en las medianías del Norte y cumbres. Es el caso de Los Rodeos, donde los episodios lluviosos de un día suponen el 39\% y tan sólo acumulan el 7\% de la precipitación anual (fig. 7). La nubosidad propia de los alisios es la responsable de ese elevado número de días con lloviznas que, sin embargo, aportan mucha menos agua que los frentes de las borrascas atlánticas. Éstas descargan precipitaciones a lo largo de dos o más días y en ellos se recogen cantidades considerables de agua. Es, precisamente, en las localidades expuestas a esos frentes donde se dan las rachas lluviosas más largas, llegando a superarse los 20 días consecutivos lloviendo. Ejemplos de ello son Sabinosa (23 días: del 28 de octubre al 19 de noviembre de 1983) y los Rodeos (22 días: del 30 de diciembre de 1977 al 20 de enero de 1978); en ambos casos se recogieron $228,6 \mathrm{~mm}$ y $226,9 \mathrm{~mm}$ respectivamente.

\section{Figura 7} RELACIÓN ENTRE LA DURACIÓN DE LOS EPISODIOS LLUVIOSOS Y LA CANTIDAD DE LLUVIA RECOGIDA EN EL
AEROPUERTO DE LOS RODEOS (TENERIFE)

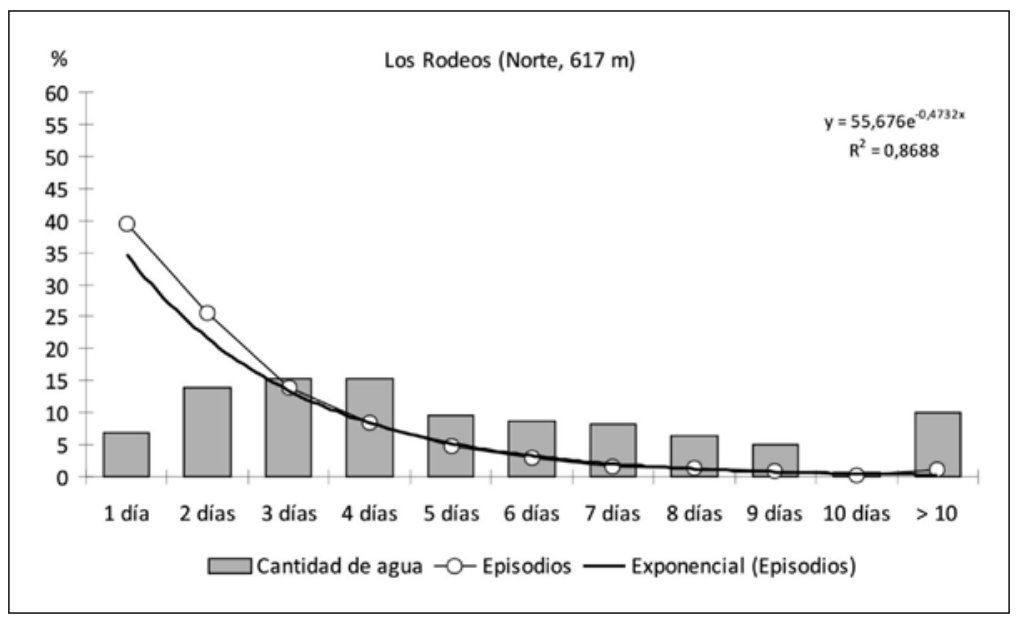

El análisis de detalle de las rachas lluviosas enseña otra característica de la concentración de la precipitación en los sectores de cumbres insulares, y es que, a pesar de que los episodios lluviosos tienen una duración entre tres y cuatro días, la mayor parte de la lluvia cae en uno o dos, coincidiendo con el paso del frente sobre las islas (figura 8). Un ejemplo de ello es el episodio de ocho días lluviosos, del 3 al 10 de diciembre de 1991, en Cuevas Blancas (Gran Canaria), en el que se registraron 660,2 mm; ahora bien, el 96\% de esa lluvia se produjo en los tres primeros días, cantidad que supuso el $85 \%$ de la lluvia de ese mes y el $57 \%$ del total anual.

Tomando como ejemplo dos localidades de Gran Canaria, Valleseco y San Bartolomé de Tirajana, situadas en vertientes opuestas, Norte-Sur, pero a altitudes semejantes (960 y 887 m.s.n.m. respectivamente) se puede observar el desigual comportamiento interanual de la lluvia en lo que respecta a la frecuencia de las rachas de uno y dos días y a la cantidad de lluvia que suponen esos días en el monto total anual (fig. 9). A pesar de la variabilidad 
Figura 8

RELACIÓN ENTRE LA DURACIÓN DE LOS EPISODIOS LLUVIOSOS Y LA CANTIDAD DE LLUVIA RECOGIDA EN CUEVAS BLANCAS (GRAN CANARIA)

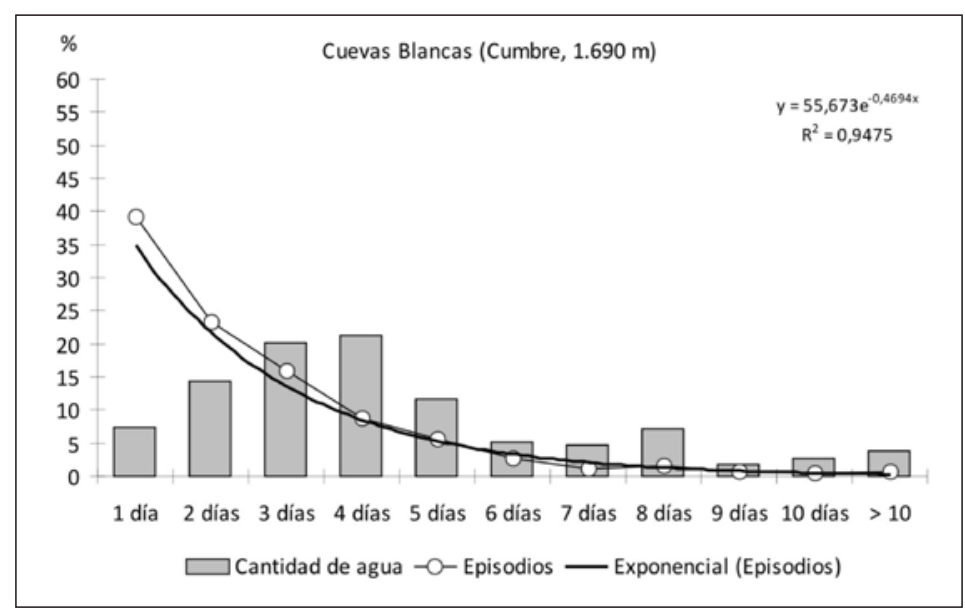

Figura 9

FRECUENCIA INTERANUAL DE LAS RACHAS LLUVIOSAS DE UN DÍA DE DURACIÓN Y CANTIDAD DE AGUA QUE SUPONEN EN EL TOTAL ANUAL EN DOS LOCALIDADES DE GRAN CANARIA SITUADAS EN VERTIENTES OPUESTAS
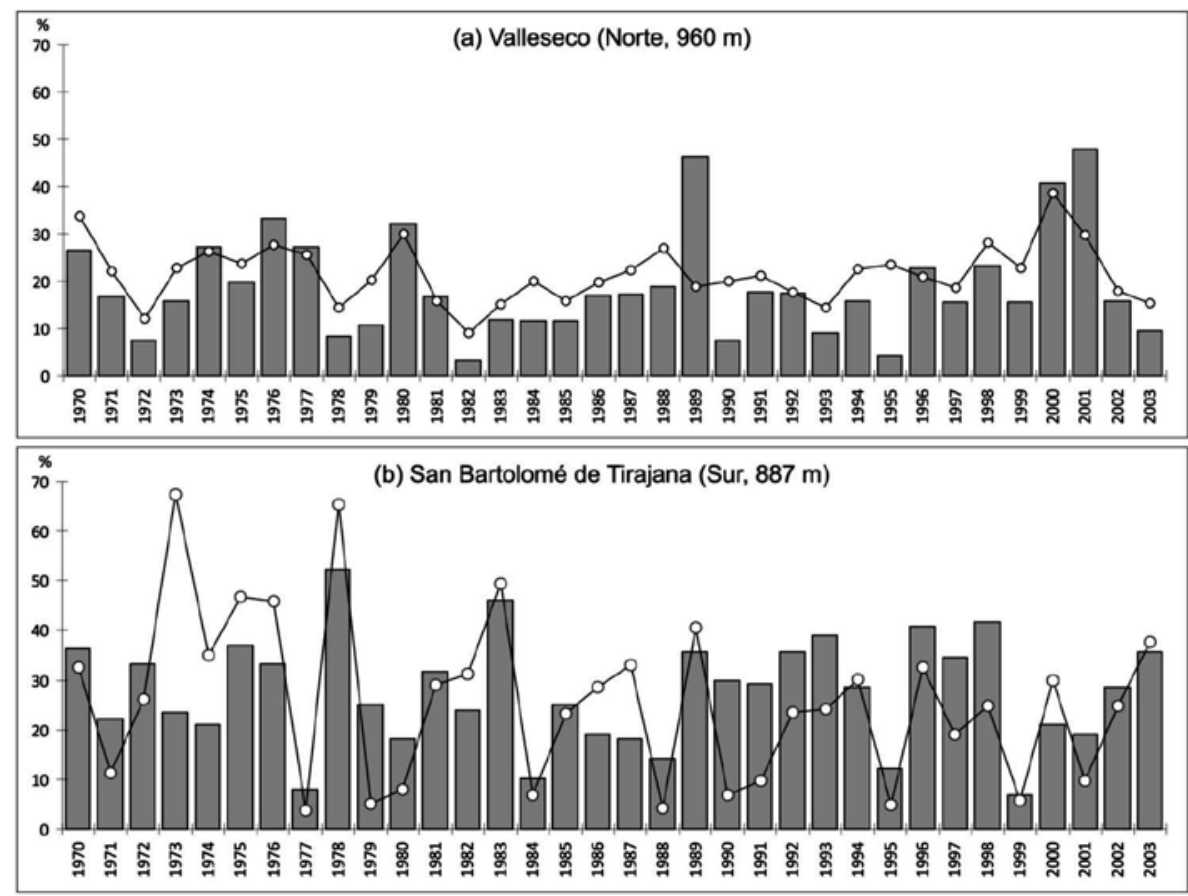

$\square$ de rachas de un día $-0-\%$ de agua sobre el total anual 
interanual, los días lluviosos aislados son bastante más frecuentes en la vertiente meridional que en la septentrional y aportan más lluvia al total anual. De media, el 30\% de los días con precipitación se producen en episodios aislados de un solo día en San Bartolomé de Tirajana frente a la mitad, el 15\%, en Valleseco, y esos días suministran el 15\% y el 10\% del total de lluvia en ambas localidades.

En lo que se refiere a las rachas de dos días consecutivos lloviendo, las diferencias entre ambas vertientes se acortan, así en el Sur su frecuencia media es del $28 \%$ y aportan el $25 \%$ de la precipitación total anual mientras que en la orientada al Norte representan el $22 \%$ de los episodios lluviosos y suponen el $20 \%$ de toda el agua caída en el año. Hay que señalar la importancia que adquieren estas rachas de dos días en aquellos años secos o muy secos como, por ejemplo, 1973 y 1978. En esos dos años en San Bartolomé de Tirajana llovió $119,1 \mathrm{~mm}$ y $156,8 \mathrm{~mm}$, menos de la mitad de lo habitual. La lluvia caída en esos años lo hizo en un reducido número de días (17 y 23 respectivamente) siendo, en ambos casos, las rachas de dos días las que más aportaron al total anual (el 65\%). En Valleseco el año 2001 fue uno de los más secos de los últimos 50 años y en los 41 días de precipitación sólo se recogieron 254,9 mm, casi un tercio de lo habitual; en este caso las 14 rachas de dos días acumularon la mitad de toda la lluvia caída ese año (fig. 10).

Figura 10

FRECUENCIA INTERANUAL DE LAS RACHAS LLUVIOSAS DE DOS DÍAS DE DURACIÓN Y CANTIDAD DE AGUA QUE SUPONEN EN EL TOTAL ANUAL EN DOS LOCALIDADES DE GRAN CANARIA SITUADAS EN VERTIENTES OPUESTAS
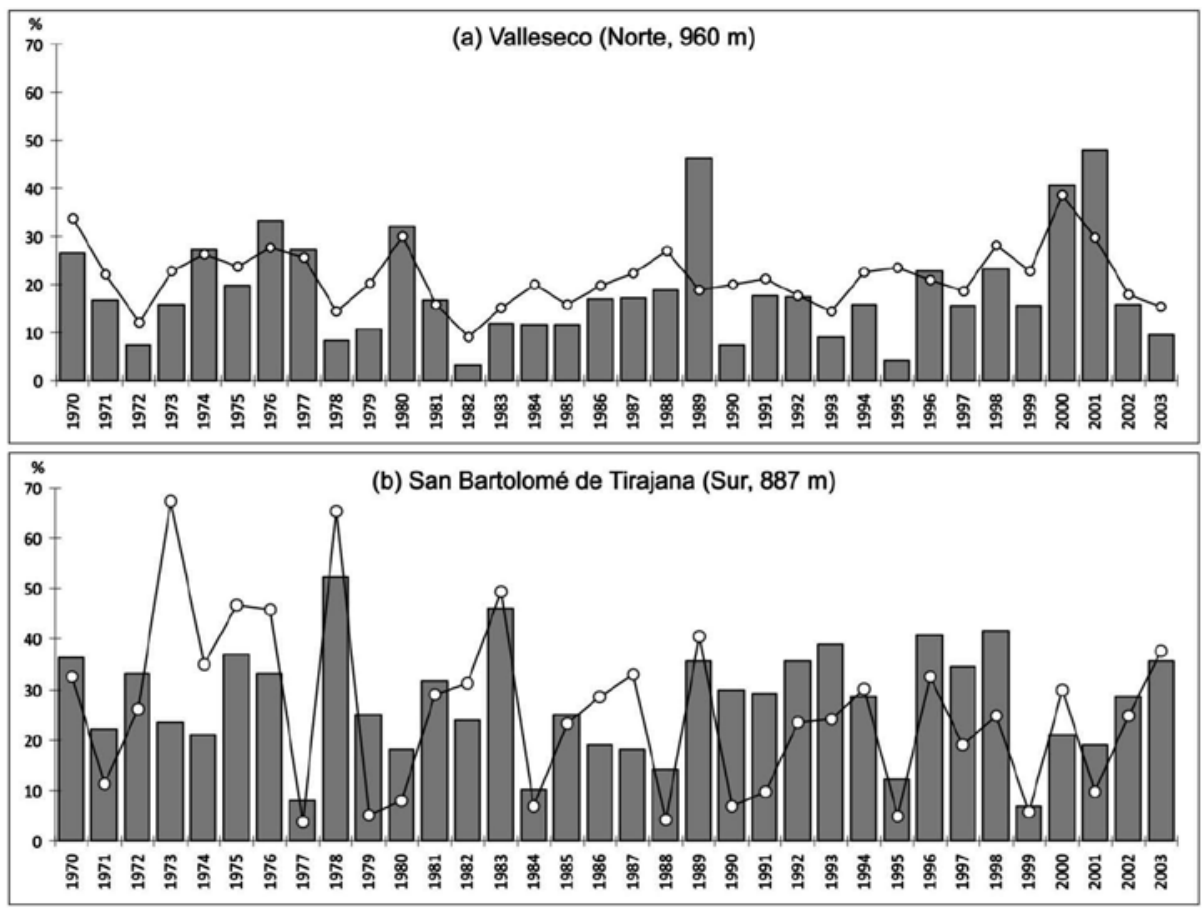

$\square \%$ de rachas de un dia $-\infty \%$ de agua sobre el total anual 


\section{CONCLUSIONES}

El análisis diario de la precipitación permite asegurar que uno de los rasgos más característicos de la pluviosidad en Canarias es su elevada irregularidad y concentración pluviométrica. Los valores moderadamente altos del CI así lo demuestran e indican que en pocos días lluviosos se acumula un porcentaje muy significativo de toda la precipitación anual. El origen de esa precipitación está vinculado a la llegada hasta el archipiélago canario de las masas nubosas procedentes de las perturbaciones atlánticas, preferentemente durante los meses de diciembre y enero. Ahora bien, esa irregularidad de la lluvia no es igual en todas las islas, ni tan siquiera en el conjunto de una sola de ellas; de manera que en todo análisis que se haga de esta variable climática es necesario considerar los factores de la orientación y altitud del relieve así como la localización de cada isla en el conjunto del archipiélago.

Así, las islas más orientales y con menor altitud, Lanzarote y Fuerteventura, tienen los índices CI más bajos, entre 0,63 y 0,65. Ello supone que el 25\% de los días más lluviosos aportan el $75 \%$ de la lluvia total. En estas islas, las lluvias de un solo día de duración son muy frecuentes y acumulan cantidades considerables con respecto al total anual, hasta el $25 \%$.

En las restantes islas es necesario diferenciar entre las vertientes meridionales, donde se registran los valores del CI más altos, del orden de 0,70 , y las septentrionales, en las que este índice es algo más moderado, entre 0,63 y 0,66. En este caso el $25 \%$ de los días más lluviosos aportan, aproximadamente, el $75 \%$ de toda la lluvia.

El factor de la altitud no introduce cambios significativos en el CI, sin embargo sí lo hace en lo referente a la duración de las rachas lluviosas. Así, en las medianías septentrionales, las lluvias se reparten en un elevado número de días lluviosos que dejan cantidades poco significativas sobre el total anual pues se tratan, en la mayoría de los casos, de lloviznas causadas por el estancamiento del mar de nubes a esa altitud. En esas vertientes también se producen fuertes aguaceros, causados por los frentes fríos atlánticos que, con mayor probabilidad, barren Canarias desde finales del otoño y durante el invierno, descargando importantes cantidades de agua en rachas de varios días lluviosos seguidos, y que incrementan considerablemente los totales anuales. En cambio, en las medianías orientales y meridionales, los valores del CI son altos, siempre superiores a 0,68. En esas vertientes se añade otro factor de importante peligrosidad como es el carácter torrencial de la precipitación.

\section{BIBLIOGRAFÍA}

AROZENA, M. E. (1993): «Los factores explicativos del paisaje vegetal», en Geografía de Canarias, vol. I, 149-164.

CEBALLOS, L. y ORTUÑO, F. (1976): Estudio sobre la vegetación y flora forestal de las Canarias Occidentales. Santa Cruz de Tenerife. Cabildo Insular de Tenerife.

COSCARELLI, R. y CALOIERO, T. (2012): «Analysis of daily and monthly rainfall concentration in Southern Italy (Calabrian region)». Journal of Hydrology, n' 416-417, 145 156.

DÁVILA, P. y ROMERO, L. (1993): «Precipitaciones máximas en Lanzarote: régimen de intensidades y frecuencias» en V Jornadas de Estudios sobre Fuerteventura y Lanzarote, tomo II, 54-72. 
DE LUIS, M., GONZÁLEZ-HIDALGO, J.C., RAVENTÓS, J., SÁNCHEZ, J.R., y CORTINA, J. (1997): «Distribución espacial de la concentración y agresividad de la lluvia en el territorio de la comunidad valenciana». Cuaternario y Geomorfología, $\mathrm{n}^{\circ}$ 11, 33-44.

DE LUIS, M., RAVENTOS, J., GONZALEZ-HIDALGO, J.C., SANCHEZ, J.R., y CORTINA, J. (2000): «Spatial analysis of rainfall trends in the region of Valencia (East of Spain)». International Journal of Climatology, n 20, 1451-1469.

DE LUIS, M., GONZALEZ-HIDALGO, J.C., BRUNETTI, M., y LONGARES, L.A. (2011): «Precipitation concentration changes in Spain 1946-2005». Natural Hazards and Earth System Sciences, $\mathrm{n}^{\mathrm{o}}$ 11, 1259-1265.

DORTA, P. (2007): «Catálogo de riesgos climáticos en Canarias: amenazas y vulnerabilidad». Geographicalia, no $51,133-160$.

HORCAJADA, T., SIMANCAS, M. y DORTA, P. (2000): «La constatación y validación de los mapas de riesgo de avenidas en pequeñas cuencas hidrográficas mediante SIG. Propuesta metodológica y aplicación a la ordenación del territorio». Boletín de la AGE, $\mathrm{n}^{\circ} 30,135-154$.

HOUGHTON, J.T., DING, Y., GRIGGS, D.J., NOGUER, M., VAN DER LINDEN, P.J., DAI, X., MASKELL, K., y JOHNSON, C.A. (2001): Climate change 2001: The Scientific Basis, Contribution of working Group I to Third Report of the Intergovermental Panel on Climate Change. Cambridge University Press.

IPCC, (2012): «Managing the Risks of Extreme Events and Disasters to Advance Climate Change Adaptation» en A Special Report of Working Groups I and II of the Intergovernmental Panel on Climate Change (Field, C., Barros, V., Stocker, T., Qin, D., Dokken, D., Ebi, E., Mastrandrea, M., Mach, K., Plattner, G., Allen, S., Tignor, M. y Midgley, P, Eds.). UK, Cambridge University Press.

KARL, T.R. y KNIGHT, R.W. (1998): «Secular trends of precipitation amount, frequency, and intensity in the United States». Bulletin of the American Meteorological Society, $\mathrm{n}^{\circ}$ 79 (2), 231-241.

LI, X., JIANG, F., LI, L. y WANG, C. (2011): «Spatial and temporal variability of precipitation concentration index, concentration degree and concentration period in Xinjiang, China». International Journal of Climatology, $\mathrm{n}^{\text {o }} 31$, 1679-1693.

MARTÍN, J.L., BETHENCOURT, J. y CUEVAS, E. (2012) «Assessment of global warming on the island of Tenerife, Canary Islands (Spain). Trends in minimum, maximum and mean temperatures since 1944». Climatic Change, $\mathrm{n}^{\circ}$ 114, 343-355.

MARTÍN-VIDE, J. (2004): «Spatial distribution of a daily precipitation concentration index in peninsular Spain». International Journal of Climatology, $\mathrm{n}^{\circ} 24,959-971$.

MARZOL, M.V., YANES, A., ROMERO, C., BRITO DE AZEVEDO, E., PRADA, S. y MARTINS, A. (2006): «Los riesgos de las lluvias torrenciales en las islas de la Macaronesia (Azores, Madeira, Canarias y Cabo Verde», en Clima, Sociedad y Medio Ambiente (Cuadrat, J.M., Saz, M.A., Vicente, S.M., Lanjeri, S. De Luis, A. y González-Hidalgo, J.C. Eds.). Zaragoza, Asociación Española de Climatología, serie A, nº 5, 443-452.

MARZOL, M.V. (1988): La lluvia, un recurso natural para Canarias. Santa Cruz de Tenerife. Caja General de Ahorros de Canarias.

MÁYER, P. (2011): Lluvias e inundaciones en Gran Canaria, Islas Canarias, España. Madrid. Editorial Académica Española. 
MÁYER, P., PÉREZ-CHACÓN, E. (2006): «Tourist activity and floods on the southern coast of Gran Canaria. An induced risk?». Journal of Coast Research, $\mathrm{n}^{\circ}$ 48, 77-80.

ROMERO, M.C., YANES, A. y MARZOL, M.V. (2004): «Caracterización y clasificación de las cuencas y redes hidrográficas en islas volcánicas atlánticas (Azores, Madeira, Canarias y Cabo Verde)» en IV Congreso Ibérico sobre Gestión y Planificación del agua. Tortosa.

SÁNCHEZ, J., RÍOS C., PÉREZ-CHACÓN, E. y SUÁREZ, C. (1985): Cartografía del potencial del medio natural de Gran Canaria. Las Palmas de Gran Canaria. Cabildo Insular de Gran Canaria, Universidad de Las Palmas de Gran Canaria y Universidad de Valencia.

SÁNCHEZ-LORENZO, A. y MARTÍN-VIDE, J. (2006): «Distribución espacial de la concentración pluviométrica diaria en la península Ibérica». Proceedings $5^{a}$ Asamblea Hispano Portuguesa de Geodesia y Geofísica (CD-ROM).

ZHAI, P.M., ZHANG, X.B., WAN, H. y PAN, X.H. (2005): «Trends in total precipitation and frecuence of daily precipitation extremes over China». Journal of Climatology, $\mathrm{n}^{\circ} 18$, 1096-1108.

ZHANG, Q., XU, C.Y., MARCO, G., CHEN, Y.P. y LIU, C.L. (2009): «Changing properties of precipitation concentration in the Pearl River basin. China». Stoch Environ Res Risk Assess, ${ }^{\circ} 23,377-385$. 
